\title{
The Prevention and Treatment of Stoma Complications. A Report of 152 Cases
}

\author{
Warda Mohay Uddin ${ }^{1}$, Sami Ullah ${ }^{2}$, Zafar Iqbal $^{3}$, Wang Jie, Irfan Ullah ${ }^{4}$, \\ Wang Sun ${ }^{5}$, Tang Dong ${ }^{6}$, Wang Daorong* \\ Department Of General Surgery, Clinical Medical College, Yangzhou University \\ (Subei People's Hospital Of Jiangsu Province), Yangzhou,P.R. China \\ Corresponding author:Daorong Wang, PhD, MD, Department of Gastrointestinal Surgery, Subei People's \\ Hospital of Jiangsu Province (The Clinical Medicine College of Yangzhou Medical University), \\ Yangzhou 225001, P. R. China.
}

\begin{abstract}
:
Background: Stomal complications are prevalent and associated with a worse quality of life and increased health-economic burdens.Stoma is an opening for fecal diversion. The aim of our study was to determine the prevention and treatment of stoma complications.

Methods: This is a prospective study was carried out in a surgical unit of Subei Hospital, Yangzhou Medical College, from January, 2013 to December, 2015. Data was collected by meticulous history taking including age, gender, indication, type of stoma, type of surgery, careful clinical examination, appropriate operative findings and follow up of the cases. The results were collected, analyzed and compared with other studies.

Result: A total of 152 patients were evaluated age ranged between 21 - 90 years. Majority (60.52\%) of patients were males. The most common type of stoma made was End colostomy 121 (79.60\%) followed by loop colostomy 8 (5.26\%), loop ileostomy 24 (15.78\%) and end ileostomy 7 (4.60\%). Main indication for a stoma formation was colorectal cancers 62(40.78\%), followed by colon cancer 30 (19.73\%), and intestinal obstruction24 (15.78\%).Thirty five patients (25\%) had stoma complications. Postoperative at ward review, the most common complication in all stoma was Skin irritation which was seen in 14 (9.21\%) patients. Complications were seen more in Colostomy as compared to ileostomy. The most common stoma site was right lower abdomen (84\%), followed by left iliac fossa (13\%), right upper quadrant (2\%) and left upper quadrant $1 \%$.

Conclusion: In the construction of an intestinal stoma extreme care should be taken to avoid all situations associated with risks for future stomal complications. Main complications included local skin problems, wound infection and retraction. End colostomy is associated with highest complications.In the constructionof an intestinal stoma extremecare should be taken to avoid allsituations associated with risks for futurestomal complications.
\end{abstract}

Keywords:Stoma, prevention, Colostomy, Ileostomy, treatment, complications.

\section{Introduction}

The word "Stoma" comes from the Greek word meaning mouth or opening. (1) An intestinal stoma is an opening of the intestine on anterior abdominal wall made surgically. (2)Stomas are used to divert the fecal stream away from distal bowel in order to allow a distal anastomosis to heal as well as to relieve obstruction in emergency situation. It may be temporary or permanent; depending on their role. (3) Though a lifesaving procedure, it may result in significant number of complications. Complications are divided into early complications (up to 30 days after operation) and late complications (more than 30 days after operation). Littre of Paris was the first to make a ventral colostomy in 1710 for a baby with imperforate anus.(4) An ileostomy was first advocated in ulcerative colitis in 1912 but was not widely used until Brooke demonstrated his everted ileostomy in 1952.(5) Various Indications for which intestinal stomas are formed: ulcerative colitis, bowel obstruction, cancer of colon \& rectum, crohn's disease, congenital bowel defects, uncontrolled bleeding from large intestine, injury to the intestinal tract, inflammatory bowel disease, ischemic bowel disease, carcinoma urinary bladder and spinal cord injury.(6)

It is estimated that half of all patients with intestinalstomas will have complications. However, several studiesdescribe the rates of $70 \%-80 \%$, or even $96 \%$ during the 3 weeks after surgery. This wide range makes itdifficult to know the incidence due to factors such asfollow-up duration, definition of complications, stomatype, type of surgery (emergency or deferred), or underlying disease(4)

Stoma, though it is a lifesaving procedure, it carries significant number of complications. Despite extensive surgical expertise, complications after stoma creation still occur and often cause social isolation and a 
significant reduction in the quality of life. Factors affecting type and frequency of complications include surgical specialty, surgeon experience, emergency V Selective creation, appropriate preoperative marking and education, and patient issues such as age, obesity, diabetes and ability to care for stoma.(7)

Ileostomy/colostomy is a high medical priority in this era of stringent financial budgeting and every attempt should be made to close it as early as possible. This is especially needed if complication like leak around the appliance, skin excoriation, prolapse and problems with its high output occurs (8). In developing countries, poor nutrition and problems with the unreliable supply of stoma collecting appliances (i.e. colostomy bags) is a very tempting reason for its earlyClosure (9). The aim of our study was to determine, stoma complicationprevention and treatment.

\section{Patients and Method}

In our study a total of 152 patients was carried out in surgical unit of Subei Hospital, Yangzhou Medical College, from January, 2013 to December, 2015. All patients were admitted through emergency and OPD basis and underwent surgery for various reasons and were followed up to note any complication which resulted in the creation of intestinal stomas, and who fit in to inclusion criteria. Data was collected by meticulous history taking including age, gender, indication, type of stoma, type of surgery, careful clinical examination, appropriate operative findings and follow up of the cases. The results were collected, analyzed. All patients who underwent elective and emergency intestinal stoma construction for any underlying cause were included in the study. All patients less than 21 years, patients with urinary diversion procedures which involve creation of intestinal stomas and patients with physiological and biochemical complications were excluded from the study.

\section{Result}

Stoma formation: A total of 152 patients were included in the study. The maximum number of patients was in the age group of 60-80years. $(\mathrm{n}=70)$. Average age in study group is 47 years (ranges 21-90). 86 patients were male and 54 were Female. The average age for women was 42 years, ranging from 21 to 65 years, and that of men 49 years (table 1). There were 31 cases of ileostomy and 121 cases of colostomy in the study. About $58(42 \%)$ stomas were made in emergency and $82(58 \%)$ in main operating theatres. Hospital stay ranged from $10-62$ days.

Table-1. Age wise distribution of intestinal stomas(n=152), Male(92), Female(60)

\begin{tabular}{|lll|}
\hline Age Group (Years) & Patients & Percentage \\
$21-40$ & 12 & 7.89 \\
$41-60$ & 48 & 31.57 \\
$61-80$ & 77 & 50.65 \\
More Than 80 & 15 & 9.86 \\
\hline
\end{tabular}

73 out of 152 patients had previous comorbid conditions like Hypertension/ Diabetes/ Tuberculosis/Hepatitis/. Most of the patients (94\% of the total) were febrile $(65 \%)$ and had pedal edema (54\%), clubbing (15\%) or lymphadenopathy $(5 \%)$. Most of the patients had pallor $(68 \%)$. Low blood protein $(<4 \mathrm{gm} / \mathrm{dl})$ and low albumin level $(<3.5 \mathrm{gm} / \mathrm{dl})$ was present in $47 \%$ and $51 \%$ patients respectively.

The most common stoma constructed was colostomy 121 (79.60\%), with End colostomy being the most common subtype of colostomy performed (113 out of 121 colostomy). loop colostomy was done in 8 patients. ileostomy was done in 31 patients, out of which 24 were loop ileostomies, 7 end ileostomies. The most common stoma site was right lower abdomen (84\%), followed by left iliac fossa (13\%), right upper quadrant (2\%) and left upper quadrant $1 \%$ (table 2).

Table- 2: Types of stoma that were closed.

\begin{tabular}{|lccc|}
\hline Procedure & \multicolumn{2}{c|}{ Patients } & Percentage \\
Colostomy121 & $\mathbf{7 9 . 6 0}$ & & \\
End colostomy & & 113 & 74.34 \\
Loop colostomy & 8 & 5.26 \\
Ileostomy 31 20.39 & & & \\
Loop ileostomy & 24 & & 15.78 \\
End ileostomy & 7 & & 4.60 \\
Procedure & & & 18.57 \\
Hartman's & 26 & & 37.85 \\
Miles & 59 & 2.14 & \\
Dixon & 4 & \\
\hline
\end{tabular}


Most common indication of stoma formation was colorectal cancers 62(40.78\%), followed by colon cancer $30(19.73 \%)$, intestinal obstruction $24(15.78 \%)$, gangrenous sigmoid volvulus 13(8.55\%), abdominal injuries $9(5.92 \%)$, Rectal prolapsed 8 (5.26\%), while Colon obstruction was $6(3.94 \%)$ which is summarize inTable 3.

\begin{tabular}{|l|l|l||}
\hline \multicolumn{2}{|l|}{ Table 3: Common indication for performing the stoma } \\
\hline \hline Indication & No. & Percent \\
\hline \hline colorectal cancers & 62 & 40.78 \\
colon cancer & 30 & 19.73 \\
intestinal obstruction & 24 & 15.78 \\
gangrenous sigmoid volvulus & 13 & 8.55 \\
abdominal injuries & 9 & 5.92 \\
Rectal prolapsed & 8 & 5.26 \\
While Colon obstruction & 6 & 3.94 \\
\hline
\end{tabular}

\section{Stoma complications:}

A total of $(25 \%)$ patients had stoma complications. Postoperative at ward review, The most common complication in all stoma was Skin problems which were seen in $14(9.21 \%)$ patients However, it was more common in patients with colostomy which $(5.26 \%)$, where $(3.94 \%)$ were in ileostomy group, other complication including Parastomal hernia (3.94\%), of this where colostomy were $(3.28 \%)$ while ileostomy were $(0.65 \%)$, six $(3.94 \%)$ patients had a wound infection which is more common in colostomy $(2.63 \%)$, total of four $(2.63 \%)$ patients have Mucocutaneous separation, Fistula of stoma were (1.97), Hemorrhage (1.31\%), and $(0.65 \%)$ patients have Ischemia, stenosis and skin prolapse respectively. Complications were seen more in Colostomy as compared to ileostomy (Table 4). Peristomal hernia appeared only in patients with a colostomy. There were five peristomal hernia after 6 months and after 2 years. There were no greater complication rates seen in patients with abnormal BMI.

Table 4: Different complications of stoma in Ileostomy \& colostomy.

\begin{tabular}{llll}
\hline Complications & $\begin{array}{l}\text { Total } \\
\text { patients }\end{array}$ & Colostomy 121 & Ileostomy 31 \\
Skin Problems & 14 & $8(5.26 \%)$ & $6(3.94 \%)$ \\
Mucocutaneous separation & 4 & $3(1.97 \%)$ & $1(0.65 \%)$ \\
Skin prolapse & 1 & - & $1(0.65 \%)$ \\
Parastomal hernia & 6 & $5(3.28 \%)$ & $1(0.65 \%)$ \\
Wound infection & 6 & $4(2.63 \%)$ & $2(1.31 \%)$ \\
Fistula of stoma & 3 & $2(1.31 \%)$ & $1(0.65 \%)$ \\
Hemorrhage & 2 & $1(0.65 \%)$ & $1(0.65 \%)$ \\
Ischemia & 1 & - & $1(0.65 \%)$ \\
Stenosis & 1 & $1(0.65 \%)$ & - \\
\hline
\end{tabular}

Complications were seen more in colostomy as compared to other stoma types. Most common complications of End colostomy were skin problems which were seen in $8(5.26 \%)$ patients, parastomal hernia 5(3.28\%), wound infection4(2.63\%). End colostomy seemed to have more complication than loop colostomy. The most common stoma site was right lower abdomen $(84 \%)$, followed by left iliac fossa (13\%), right upper quadrant $(2 \%)$ and left upper quadrant $1 \%$.

\section{Discussion}

Fecal diversion remains an effective option to treat a variety of gastrointestinal and abdominal conditions (10). Ileostomy and colostomy are commonly made intestinal stomas in surgery. The first surgical stoma was created more than 200 years ago. The earliest stomas were actually unintentional ones, enterocutaneous fistulas resulting from penetrating abdominal injuries or complications of intestinal diseases such as incarcerated hernias (11). A number of patients undergo surgeries for fecal diversion. But despite a great number of such surgeries done, complications are almost inevitable.

Patients undergoing stoma formation are at risk of developing a wide range of complications following surgery (12). There are many factors suggested to predispose to stoma complications like high body mass index, 
inflammatory bowel diseases, use of steroids and immunosuppressant drugs, diabetes mellitus, old age, emergency surgery, surgical technique and surgeons' experience (13).

In our study done on 152 patients, 32 ileostomies and 120 colostomies were performed, while in a similar study by Hellman J et al (14) reported 93 patients with 35 ileostomy and 58 colostomy formation. In our study mean age was 47 years (range 21 to 90 year), while in a similar study by Nastro P et al (15) mean age was 45 years and in Cheape JD et al (16) mean age was 46 years (range 11 to 88 ).

End colostomy was the most common stoma formed (73.68\%) followed by loop colostomy $(5.26 \%)$. Colostomy accounted for 70\% stomas in another study by Ghazi MA et al (17) followed by ileostomy in 30\%. In a study by Safirullahetal (18) End colostomy was formed in $43 \%$ cases and loop colostomy in $17.4 \%$ cases. In our study number of colostomies were more as compared to ileostomies which corresponds to a study in literature (19) but in other studies number of ileostomies were more $(15,16)$.

The most common indication of stoma formation in our study was colorectal cancers $61(43.57 \%)$ cases followed by colon cancer $29(20.71 \%)$, intestinal obstruction $23(16.42 \%)$, gangrenous sigmoid volvulus 12(8.57\%), abdominal injuries 7 (5\%), Rectal prolapsed 5 (3.57\%), while Colon obstruction was $3(2.14 \%)$. Study of Akram Rajput et al (20) reported colorectal cancers (60\%) as the commonest indication of stoma formation. Adnan Aziz et al (21) demonstrated colorectal cancers (66\%) followed by tuberculosis as the most common indication. In contrast, a study of Safirullah et al (18) showed colorectal carcinoma (22\%) as the most common indication followed by trauma (20\%) and typhoid perforation $(20 \%)$.

As already discussed that intestinal stoma formation is lifesaving surgical procedure in certain situations like for example in emergency surgery where we have to deal polytrauma patients and diseased gut in such circumstances definitive bowel repairs are disastrous moreover it further prolongs operative time which further jeopardizes patients physiology. But like other surgical procedures stoma formation may also results in local, systemic and psychological complications $(12,22)$.

In our study, $75 \%$ cases remained free of complications while $25 \%$ cases developed some complication. This percentage is near to western studies by Pearl (23), Duschesne (24) and Harris (25) who reported complications in $26 \%, 25 \%$ and $25 \%$ cases respectively. The early reported incidence of peristomal skin irritation ranges from $3-42 \%$. The degree of irritation ranges from mild peristomal dermatitis to full thickness skin necrosis to ulceration.

The most common complication reported in our study was peristomal skin irritation and erythema (23\%) followed by diarrhea (15\%), mucosal prolapse and retraction (13\%), wound infection (11\%), mucocutaneous separation (10\%), Parastomal hernia (8\%), stenosis (4\%), faecal fistula (4.8\%). Bleeding (5\%), Stoma retraction $(2.5 \%)$, and necrosis $(1.5 \%)$. A study by Ratliff et al (26) has shown peristomal irritation in $33 \%$ cases while Pearl et al (23) showed peristomal skin erythema as the most common complication in $42 \%$. AmbreenMuneer (27) reported skin excoriation in $18 \%$ cases. In this study complications were seen more in Colostomy as compared to ileostomy. Similarly in a study by Park et al (23) reported highest incidence of complications in Colostomy (57\%) but Katia et al (28) reported higher overall complication rate with ileostomy.

\section{Prevention}

Surgical technique:Surgical technique (i.e., stoma protrusion, suturing) plays a critical role in preventing peristomal skin complications. Stoma protrusion has been identified as a risk factor for compromised skin because flush and retracted stomas discharge effluent at or below skin level. This increases the risk of effluent eroding the skin barrier adhesive. A stoma with adequate protrusion (i.e., $2.5 \mathrm{~cm}$ ) and a lumen pointed upward rather than bowing downward provides a spout to discharge effluent directly into the pouching system (29).

Post-op care: Postoperatively, most peristomal skin complications can be minimized or avoided when patients understand the optimal environment for healthy skin and utilize that information during care. Not all peristomal skin complications are preventable; some are disease-related, immunologic, or infectious. However, knowledge and application of basic skin care approaches are key to keeping the skin clean, dry, and protecting it from exposure to effluent, trauma, chemical injury (30).

Parastomal Hernia: This is one of the most studied technical aspects. Recent randomized trial, (31)a met analysis (32) and systematic reviews (33) observed that the incidence of parastomal hernia for both colostomy and ileostomy and in open and laparoscopic surgery was very significantly reduced using a prophylactic mesh, with no increase in complications. In their study the complication observed with prophylactic mesh is $12.5 \%$ and without mash it was 53\% (34). Furthermore, in the case of hernia, the need for surgery is $0 \%$ if a mesh is used and $13 \%$ if none is used.

Although the use of prophylactic mesh used in the same surgical procedure to construct the stoma may reduce the risk of parastomal hernia, (35)the technique or type of mesh which obtain the best results, as well as the patient groups with risk factors who could benefit are yet to be determined. 


\section{Conclusion}

In the construction of an intestinal stoma extreme care should be taken to avoid all situations associated with risks for future stomal complications. Stoma complications are incessant and compromise quality of life. Many are secondary to stoma development, and may hence be avoided through great surgical technique employed by experienced surgeons. The use of meshes reduces the risk of parastomal hernia and the rate of recurrence after repair. The role of the stoma treatment nurse is essential in selecting the stoma site in the preoperative stage and furthermore in the subsequent stage, to help ostomy patients adjust better and pick up independence, and accomplish a better quality of life.Stoma related complications can be decreased by continuous monitoring, early detection and treatment. Ostomy clinic is a public health profession and requires professionalism for success.

\section{References}

[1]. Taylor P. An introduction to stomas: reasons for their formation. Nurs Times 2005;101:63-4

[2]. Irving MH, Hulme O. Intestinal stomas. Br Med J 1992; 304: 1679-81.

[3]. Saunders RN, Hemingway D. Intestinal Stomas. SurgInt 2005;71:44-7.

[4]. Khalid AM, Irshad W. Surgical history of intestinal obstruction. Specialist 1991; 8 (1): 55-60.

[5]. Cushieri A, Steele RJ, Moosa AR. Disorders of the colon and rectum. In: Cushieri A, Steele RJ, Moosa AR. Essential Surgical Practice. London. Arnold; 2002:569-626.

[6]. Crohn's and Colitis Foundation of America. 386 Park Ave. S. 17thFloor. New York. NY 10016.(800) 826-0826.

[7]. Abbas MA, Tejirian T. Laparoscopic stoma formation. JSLS 2008; 12: 159-161.

[8]. Danielsen AK1, Correa-Marinez A, Angenete E, Skullmann S, Haglind E, Rosenberg J; Early closure of temporary ileostomy-the EASY trial: protocol for a randomised controlled trial. Br Med J Open. 2011; 1:162. Edwards DP, Chisholm EM, Donaldson DR. Closure of transverse loop colostomy and loop Ileostomy. Ann R CollSurgEngl 1998; 80:33-5.

[9]. Boyden AM. The surgical treatment of diverticulitis of colon. Ann Surg 1950;132:94-109.

[10]. Abbas MA, Tejirian T. Laparoscopic stoma formation. JSLS 2008; 12: 159-161.

[11]. Kaider-Person O, Person B, Wexner SD. Complications of construction and closure of temporary loop ileostomy. J Am CollSurg 2005;201:759-73.

[12]. MeGrath A, Porrett T, Heyman B. Parastomal hernia: an exploration of the risk factors and the implication. Br J Nurs 2006;12;31721.

[13]. Arumugam PJ, Bevan L, Macdonald L, Watkins AJ, Morgan AR, Beynon J et al. A prospective audit of stomas - analysis of risk factors and complications and their management. Colorectal Dis 2003;5:49-52

[14]. Hellman J, Lago CP, Dermatologic complications in colostomy and ileostomy patients, Int J Dermatol. 1990

[15]. Mar;29(2):129-33.

[16]. Nastro P, Knowles CH, McGrath A, HeymanB,Porrett TR, Lunniss PJ, Complications of intestinal stomas, Br J Surg. 2010 Dec;97(12):1885-9. doi: 10. 1002/bjs.7259. Epub 2010 Sep 24.

[17]. Cheape JD, Hooks VH 3rd. Loop ileostomy: a reliable method of diversion, South Med J. 1994 Mar; 87(3):370-4.

[18]. Muhammad Ahmad Ghazi, Amir RiazBhutia, Hafiz Muhammad, Asif Maqbool, NaumanDawood, Nasir Mahmood. The trends and outcome of stoma procedures in abdominal surgery, Pak Journal of Med and Health Sciences 2009;3(2):106.

[19]. Safirullah, Mumtaz N, Jan MA, Ahmed S. Complications of intestinal stomas. J Postgrad Med Inst. 2005;19(4):407-11

[20]. Ahmad Q A,Saeed M K,Muneera M J, Ahmad M S,KhalidK. Indications and complications of intestinal stomas-A tertiary care hospital experience. Biomedica. 2010; 26:144-7.

[21]. Hwang YF, Chen SS, Liou TY, Wang HM, Hsu H, Complications of colostomies and colostomy closure, [Article in Chinese] Gaoxiong Yi XueKeXueZaZhi. 1990 Jun;6(6):276-82.

[22]. Adnan Aziz, Irjan Sheikh Masood Jawant, ShamsudeenAlam, ManzarSaleem, Indications and complications of loop ileostomy, Journal of surgery Pakistan (international) 2009 Jul-Sept14(3).

[23]. Szczepkowski M, Waskiewicz W, Bielecki K, Gornicki K. Local complications in ostomates. Br J Surg 1994; 81:65.

[24]. Pearl RK, Prasad ML, Orsay CP, Abcarian H, Tan AB, Melze MT Early local complications from intestinal stomas, Asch Surg $1985 ; 120$ (10):1145-47.

[25]. Duchesne JC, Wang X, Weintraub SL, Boyle M, Hunt JP, Stoma complications, multivariate analysis. Am Surg.2002;68(11):961 66.

[26]. Harris RP, Daly KJ, Jones LS, Kiff ES. Colorectal Dis 2004 jul;6(4):280-4

[27]. Ratliff, Catherino R. Journal of wound, Ostomy \& Continence Nursing 2010;37(5):505-510

[28]. AmbreenMuneer, A. Razaque Shaikh, GulsanAra Shah, Ali Qureshi, Liaquat University of Medical \& Health Sciences, Jamshoro, Sind Pakistan. World Applied Sciences Journal 2007; 2(3):190-193.

[29]. Katia Ferreira Guenaga, Suzana Angelica, Silva Lustosa. Ileostomy or Colostomy for temporary decompression of colorectal anastomosis-a systematic review and metaanalysis, Acta Cir. Bras;2008may/jun:23(3).

[30]. Gordon PH, Rolstad BS, Bubrick MP. Intestinal stomas. In: Gordon PH, Nivatvongs S, eds. Principles and Practice of Surgery for the Colon, Rectum and Anus, Edition 2. St. Louis, Mo.: Quality Medical, 1998

[31]. Erwin-Toth P. Prevention and management of peristomal skin complications. Advances in Skin \& Wound Care. 2000;13(4):175179.

[32]. Hammond TM, Huang A, Prosser K, Frye JN, Williams NS. Parastomal hernia prevention using a novel collagen

[33]. inplant: a randomized controlled phase I study. Hernia. 2008;12:475-81.

[34]. Wijeyekoon SP, Gurusamy K, El-Gendy K, Chan CL. Prevention of parastomal herniation with biologic/ composite prosthetic mesh: a systematic review and meta- analysis of randomized controlled trials. J Am Coll Surg. 2010;211:637-45.

[35]. Tam KW, Wei PL, Kuo LJ, Wu CH. Systematic review of the use of a mesh to prevent parastomal hernia. World J Surg. 2010;34:2723-9.

[36]. Shabbir J, Chaudhary BN, Dawson R. A systematic review on the use of prophylactic mesh during primary stoma formation to prevent parastomal hernia formation. Colorectal Dis. 2012;14:931-6.

[37]. Cima RR, Turnage R, Collins KA. Parastomal hernia. Up to date 2012. Available from: http://www.uptodate.com/contents/parastomal-hernia. 